\title{
Una nueva localidad para la familia Lacandoniaceae y nuevos registros para la Reserva de Montes Azules, Chiapas, México
}

El descubrimiento de una nueva localidad para la familia Lacandoniaceae es el resultado de las colecciones botánicas que realizamos como parte del proyecto multidisciplinario: "Aprovechamiento autosostenido de recursos naturales en la Selva Lacandona: abejas y flora necta-polinífera de los ecosistemas de la Región Las Cañadas, Chiapas».

El nuevo registro se localiza dentro de la Reserva de Montes Azules, cerca a la colonia Benito Juárez Miramar $\left(16^{\circ} 12^{\prime} \mathrm{N}\right.$ y $\left.91^{\circ} 13^{\prime} \mathrm{O}\right)$ (Fig. 1). Hacia el lado opuesto (al $\mathrm{NE})$ se encuentra la localidad tipo $\left(15^{\circ} 45^{\prime} 08^{\prime \prime} \mathrm{N}\right.$ y $90^{\circ} 56^{\prime}$ 00" O) (Martínez y Ramos, 1989), pero queda fuera de dicha reserva, en la zona de amortiguamiento. Con este nuevo hallazgo se amplía la distribución de la familia Lacandoniaceae que, hasta ahora, se pensaba tenía una distribución limitada a la localidad tipo.

Entre las dos localidades en las que se ha colectado Lacandonia schismatica E. Martínez et C. H. Ramos existe, aproximadamente, una distancia lineal de $52 \mathrm{~km}$. Esta separación nos hace suponer, por un lado, que la familia (monoespecífica) puede estar distribuida en este intervalo y, por otro, que ocupa diferentes tipos de vegetación. Esta última idea está apoyada por la diversidad de hábitats en los

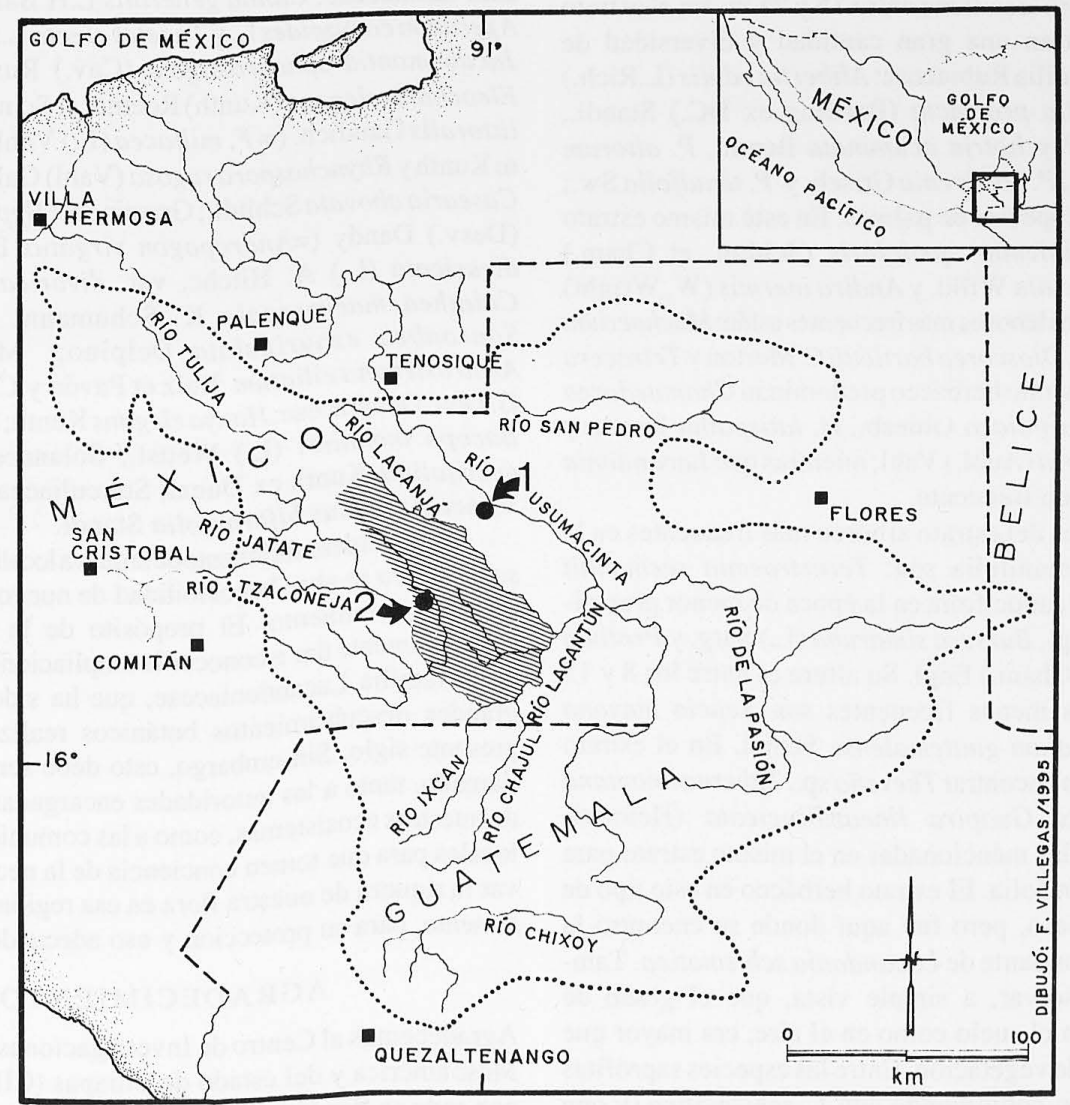

Figura 1. Localidades donde se ha colectado Lacandonia schismatica: 1 corresponde al primer registro; 2 representa la nueva localidad y está dentro de la Reserva de Montes Azules (11). La zona delimitada por puntos corresponde a la Región Lacandona (Martínez et al., 1989).

Mapa tomado de Martínez et al. (1989) y de SEDUE (1989). 
que se encontró la nueva localidad (aproximadamente una hectárea). En contraste, el primer registro de L. schismatica se hizo únicamente en un solo tipo de vegetación: selva alta subperennifolia inundable de Calophyllum brasiliense Camb., Terminalia amazonica (J. Gmelin) Exell y Cryosophila argentea Bartlett (Martínez y Ramos, 1989). Considerando los puntos anteriores, y el área de la Región Lacandona (Fig. 1) (Martínez y Ramos, 1989), no es difícil suponer que $L$. schismatica se encuentra en Guatemala.

Los tipos de vegetación en los que se observó el nuevo registro de Lacandonia schismatica son: selva alta perennifolia, selvas mediana y baja subperennifolias. La composición florística en cada una de ellas es variable, pero con frecuencia se encuentran, en mayor o menor medida, las mismas especies. Por ejemplo, en el estrato arbóreo de las selvas alta perennifolia y mediana subperennifolia son frecuentes: Blepharidium guatemalense Standl., Vochysia hondurensis Sprague, Spondias radlkoferi J.D.Smith, Bursera simaruba Sarg., Protium copal Schltdl. et Cham. y Alseis yucatanensis Standl. La altura de estas especies varía entre 25 y $40 \mathrm{~m}$ en la selva alta, mientras que en la selva mediana el mismo estrato arbóreo tiene entre 15 y $25 \mathrm{~m}$. En el estrato medio se encuentran una gran cantidad y diversidad de elementos de la familia Rubiaceae: Alibertia edulis (L.Rich.) A. Rich., Chomelia protracta (Bartling ex DC.) Standl., Chomelia spp., Psychotria acuminata Benth., P. altorum Standl. et Steyerm, $P$. costivenia Griseb. y P. tenuifolia Sw.; así como algunas especies de palmas. En este mismo estrato son frecuentes Clidemia petiolaris (Schltdl. et Cham.) Schltdl., Inga punctata Willd. y Andira inermis (W. Wright) DC. Entre los bejucos leñosos más frecuentes están: Machaerium cirrhiferum Pittier, Dioscorea bartlettii C.Morton y Tetracera volubilis L. En el estrato herbáceo predominan Chamaedorea sp., Heliconia aurantiaca Ghiesb., H. latispatha Benth. y Cephaelis tomentosa (Aubl.) Vahl, mientras que Lacandonia schismatica es poco frecuente.

Las especies del estrato arbóreo más frecuentes en la selva baja subperennifolia son: Ternstroemia seemannii Triana et Planch. (caducifolia en la época de menor precipitación), Bumelia sp., Bursera simaruba (L.) Sarg. y Protium copal (Schltdl. et Cham.) Engl. Su altura es entre los 8 y 15 m. Otras especies menos frecuentes son Acacia mayana Lund. y Blepharidium guatemalense Standl. En el estrato arbustivo es común encontrar Thevetia sp., Tabernaemontana chrysocarpa Blake, Guapira linearibracteata (Heimerl) Lundell así como las mencionadas en el mismo estrato para la selva alta perennifolia. El estrato herbáceo en este tipo de vegetación es escaso, pero fue aquí donde se encontró la población más abundante de Lacandonia schismatica. También se pudo observar, a simple vista, que el grado de humedad, tanto en el suelo como en el aire, era mayor que en los otros tipos de vegetación. Entre las especies saprófitas que se encontraron conviviendo con L. schismatica (y que coinciden con aquellas encontradas en la localidad tipo), son: Voyria parasitica (Schltdl. et Cham.) Ruyters et Maas, V. tenella W.J. Hooker y Gymnosiphon sp. En este mismo tipo de vegetación se encontraron varias especies de orquídeas terrestres: Chranichis sp., Maxillaria variabilis Batem. ex Lind. y Sobralia fragrans Lindl., entre otras.

Entre los géneros colectados se encuentran: Helicteres el cual no es registrado por Martínez et al. (1994). Por otra parte, Cordia eriostygma Pittier, Catopsis apricoides (Schltdl. et Cham.) Baker, Callisia filiformis (Martens et Galeotti) Hunt y Microgramma percusa son especies que Breedlove (1986) y Martínez et al. (1994), no registran, respectivamente, para Chiapas y la región Lacandona. Otra especie, Cipura campanulata tampoco la registran los autores anteriores, pero es incluida en la revisión que hacen Henrich y Goldblatt (1994) en el Vol. VI de la Flora Mesoamericana. También se colectaron, por vez primera: Coccoloba schiedeana Lindau, Ficus padifolia Kunth, Hibiscus costatus A.Rich., Roupala borealis Hemsl. (estéril) y Sageretia elegans (Kunth) Brong. Estas especies son incluidas en el listado de la región Lacandona (Martínez et al., 1994), pero fueron tomadas por los autores de Flora of Guatemala. Otras especies colectadas en la región y que no son registradas por Martínez et al. (1994), pero sí en el listado de Chiapas (Breeedlove, 1986) son: Cannaceae: Canna generalis L.H.Bailey; Compositae: Ageratum conyzoides L. y Tagetes erecta L.; Convolvulaceae: Jacquemontia sphaerostigma (Cav.) Rusby; Cyperaceae: Eleocharis elegans (Kunth) Roemer et Schultes, Fimbristylis littoralis Gaudich. (=F. miliacea (L.) Vahl), Fuirena robusta Kunth y Rhynchospora rugosa (Vahl) Gale; Flacourtiaceae: Casearia obovata Schltdl.; Gramineae: Hypogynum virgatum (Desv.) Dandy (=Andropogon virgatus Desv.) y Lasiacis divaricata (L.) A. Hitchc. var. divaricata; Marantaceae: Calathea macrosepala K. Schumann; Marcgraviaceae: Souroubea exauriculata Delpino; Melastomataceae: Arthrostemma ciliatum Ruiz et Pavón y Clidemia hirta (L.) G. Don; Onagraceae: Hauya elegans Kunth; Scrophulariaceae: Bacopa monnieri (L.) Wettst.; Solanaceae: Solanum cf. acerifolium Kunth ex Dunal; Sterculiaceae: Helicteres sp.; Vitaceae: Cissus biformifolia Standl.

Coneldescubrimiento de la nuevalocalidad de Lacandonia schismatica se abre la posibilidad de nuevos estudios para su mejor conocimiento. El propósito de la presente nota es principalmente dar a conocer la ampliación de la distribución de la familia Lacandoniaceae, que ha sido uno de los más grandes descubrimientos botánicos realizados al final del presente siglo. Sin embargo, esto debe servir para hacer un llamado, tanto a las autoridades encargadas de la protección de nuestros ecosistemas, como a las comunidades campesinas locales para que tomen conciencia de la necesidad de conservar la riqueza de nuestra flora en esa región y actuar, conjuntamente, para su protección y uso adecuado.

\section{AGRADECIMIENTOS}

Agradecemos al Centro de Investigaciones Humanísticas de Mesoamérica y del estado de Chiapas (CIMECH), UNAM, con sede en San Cristóbal las Casas, y en particular al Dr. J. Pohlenz Córdova, coordinador del proyecto por su invitación a participar en el mismo. También agradecemos a la SEDESO quien financió el proyecto. 


\section{LITERATURA CITADA}

Breedlove DE. 1986. Listados florísticos de México IV. Flora de Chiapas. Instituto de Biología, UNAM, México.

Henrich JE, Goldblatt P. 1994. 5. Cipura Aublet. En: Davidse G, Sousa M, Chater A, edrs. Flora Mesoamericana. Alismataceae a Cyperaceae. Vol. 6. Instituto de Biología, UNAM, Missouri Botanical Garden y The Natural History Museum (London). México, 75-76.

Martínez E, Ramos C H, Chiang F. 1994. Lista florística de la Lacandona, Chiapas. Boletín de la Sociedad Botánica de México 54: 99-177.

Martínez E, Ramos C H. 1989. Lacandoniaceae (Triuridales): una nueva familia de México. Annals of the Missouri Botanical Garden 76: 128-135.

Sedue. 1989. Información básica sobre las áreas naturales protegidas de México. Subsecretaría de Ecología. México, D. F.
ALBERTO REYES-GARCÍA Y MARIO SOUSA S. Herbario Nacional de México, Instituto de Biología, UNAM, Apdo. Postal 70-367. Coyoacán, 04510, México, D. F. 